\title{
„Entspricht der Landtag ... dem Volksbegehren“ - Probleme eines kupierten direktdemokratischen Verfahrens am Beispiel Brandenburg
}

\author{
Otmar Jung
}

\section{Der Landtag Brandenburg entspricht einem Volksbegehren}

Im Dezember 2012 war das Volksbegehren „Nachtflugverbot“1 in Brandenburg zustande gekommen. Zwei Monate später vollzog die Landesregierung, die bislang das Anliegen der Initiatoren abgelehnt hatte, einen überraschenden Schwenk. ${ }^{2}$ Nach einer Sitzung des Koalitionsausschusses von SPD und PDS verkündete Ministerpräsident Matthias Platzeck am 19. Februar 2013, man wolle das Anliegen des Volksbegehrens übernehmen. ${ }^{3}$ Der Landtag solle bereits in der folgenden Woche einen entsprechenden Beschluss fassen. Zur Begründung erklärte Platzeck, die Debatte um den Flughafen werde von extrem entgegengesetzten Positionen aus geführt: „Den Hoffnungen auf Arbeit und wachsenden Wohlstand der einen stehen Sorgen um Lärmbelastung und sinkende Lebensqualität der anderen gegenüber. Ich bin mir sicher: Ein Volksentscheid mit einer vorangegangenen monatelangen Kampagne würde die erkennbare Spaltung des Landes vertiefen. Ich will diese Zuspitzung vermeiden und den Zielkonflikt entschärfen." Ferner würde die Abhaltung eines Volksentscheids noch Zeit kosten, die er für eine Sachlösung nutzen wolle. ${ }^{4}$

Die Nachtfluggegner waren hocherfreut: „Wir sind zunächst einmal glücklich“, erklärte einer der Initiatoren des Volksbegehrens. „Die Arbeit hat sich gelohnt. Einbringen lohnt sich. “ Dies sei ein wichtiges Signal für die Demokratie. ${ }^{5}$ Der Landesverband Berlin-Brandenburg von Mehr Demokratie e.V. meinte, die Übernahme von Volksbegehren sei „erfreulich im Sinne der politischen Kultur"6.

1 So lautete die Kurzbezeichnung des Volksbegehrens „Für eine Änderung des $₫ 19$ Absatz 11 des Landesentwicklungsprogrammes zur Durchsetzung eines landesplanerischen Nachtflugverbotes am Flughafen Berlin Brandenburg International (BER)!“.

2 Dass das Volksbegehren zustande gekommen war, stand bereits nach dem Vorläufigen Ergebnis fest, vgl. Pressemitteilung (PM) des Landesabstimmungsleiters Nr. 3/12 vom 3. Dezember 2012. Das endgültige Gesamtergebnis des Volksbegehrens wurde verkündet durch Bekanntmachung des Landtagspräsidenten vom 22. Januar 2013, GVBl. I S. 1. Die Regierung und die Parteien hatten also über zwei Monate Zeit zum Nachdenken gehabt.

3 Verfassungsrechtlich ist es der Landtag, der einem Volksbegehren entsprechen kann (Art. 78 Abs. 1 Satz 1 der Verfassung des Landes Brandenburg (BbgVerf)). Politisch trifft eine solche Entscheidung der Regierungschef im Einvernehmen mit den führenden Akteuren der parlamentarischen Mehrheit. Diese Binnenstruktur ist hier jedoch nicht das Thema. Wenn die Darstellung oft auf Ministerpräsident Platzeck abhebt, ist er gleichsam als Gesicht und Verkünder einer bestimmten Politik gemeint; es soll das Problem nicht unangemessen personalisiert werden.

4 Vgl. Staatskanzlei, Neue Videobotschaft des Ministerpräsidenten: Nachtflugkonflikt entschärfen vom 20. Februar 2013, http://www.stk.brandenburg.de/cms/detail.php?gsid=bb1.c.323111.de (Abruf am 22. Februar 2013). Dies war die elaborierteste von mehreren Argumentationen. Platzecks Stellungnahme im Landtag eine Woche später wirkte bereits geglättet, vgl. Landtag (LT) Brandenburg (Bbg), Plenarprotokoll (PIPr) Nr. 5/71 vom 27. Februar 2013, S. 5769 ff.

5 Vgl. Alexander Fröhlich / Thorsten Metzner, Schöner schlafen in Schönefeld, in: Der Tagesspiegel vom 20. Februar 2013.

6 PM 7/13 vom 26. Februar 2013. 
Acht Tage nach Platzecks Ankündigung stimmte der Landtag mit großer Mehrheit einer Beschlussempfehlung des zuständigen Ausschusses zu, das Volksbegehren anzunehmen ${ }^{7}$; es gab 62 Ja- und fünf Nein-Stimmen bei 14 Enthaltungen. Die Koalitionsfraktionen SPD und Die Linke sowie die oppositionelle Fraktion Bündnis 90/Die Grünen stimmten fast geschlossen mit Ja. ${ }^{8}$ Von der CDU-Fraktion enthielten sich 14 Abgeordnete, während drei mit Ja votierten. Bei der FDP stimmten vier Abgeordnete gegen und zwei für die Übernahme. ${ }^{9}$

Dieser Übernahmebeschluss soll hier hinterfragt werden. Es geht also im Folgenden nicht um eine Diskussion des Konflikts zwischen Ökonomie (Rentabilität des Flughafens) und Ökologie (Lärmbelastung). Ebensowenig soll auf die in der Tagespolitik gestellten Fragen eingegangen werden, ob Brandenburg nach dem bestandskräftigen Planfeststellungsbeschluss aus dem Konsens mit den weiteren Gesellschaftern der Flughafen Berlin Brandenburg GmbH - Land Berlin und Bund - ausscheren und ob gerade Platzeck als Aufsichtsratsvorsitzender der Flughafen-Gesellschaft diese Wende vollziehen durfte. Untersucht werden soll vielmehr, welche Probleme ein kupiertes Verfahren der direkten Demokratie aufwirft, wie es jetzt in Brandenburg zu erleben war.

\section{2. Übernahme durch das Parlament - eine Standardvariante?}

\subsection{Vorkommen und Nutzung}

Dass das Parlament einem Volksbegehren „entsprechen“, das heißt dessen Anliegen übernehmen kann, gehört zum gemeindeutschen Landesverfassungsrecht. Die Verfassungen von 14 Bundesländern enthalten einschlägige Klauseln. In Bayern wird die Übernahmemöglichkeit durch das Ausführungsgesetz eröffnet. ${ }^{10}$ Einzig bei dem dreistufigen Verfahren in Sachsen besteht die Möglichkeit der Übernahme nur nach der ersten Stufe des Volksantrags (Art. 72 Abs. 1 Satz 1 der Verfassung), aber nicht mehr, nachdem ein Volksbegehren erfolgreich abgeschlossen wurde - der Freistaat folgt hier einer Sonderkonzeption von direkter Demokratie. ${ }^{11}$ Die Tatbestände der Regelwerke variieren im Einzelnen von „entsprechen “ und „unverändert“ oder „im wesentlichen unverändert“ übernehmen bis zu der weitestgehenden neuen Regelung in Schleswig-Holstein, dass ein Volksentscheid auch dann nicht stattfindet, wenn der Landtag dem Gesetzentwurf oder der anderen Vorlage ,in einer von den Vertreterinnen und Vertretern der Initiative gebilligten geänderten Fassung zustimmt" (Art. 42 Abs. 2 Satz 3 Nr. 1 der Verfassung).

7 Vgl. LT Bbg, Drs. 5/6894 vom 25. Februar 2013.

8 Ein SPD-Abgeordneter votierte mit Nein.

9 Abstimmungsergebnis: LT Bbg, PlPr Nr. 5/71 vom 27. Februar 2013, S. 5772 (mit Schreibversehen bei den Enthaltungen), Liste der namentlichen Abstimmung auf S. 5815. Die 62 Ja-Stimmen machten rechnerisch sogar eine Zweidrittelmehrheit des Landtags aus (88 Abgeordnete), worauf es aber rechtlich nicht ankam.

10 Vgl. Art. 73 Abs. 3 LWG.

11 Zugrunde liegt das Konzept einer „frühen Trennung von parlamentarischem und plebiszitärem Problemlösungsverfahren“ (vgl. Otmar Jung, Jüngste plebiszitäre Entwicklungstendenzen in Deutschland auf Landesebene, in: Jahrbuch des Öffentlichen Rechts der Gegenwart, 41. Jg. (1993), S. 29 - 67), das der sächsische Verfassungsgeber von der neuen Verfassung in SchleswigHolstein (1990) übernahm (S. 39): Auf ein zustande gekommenes Volksbegehren sollte automatisch ein Volksentscheid folgen. Schleswig-Holstein selbst hat 2004 wieder eine Übernahmeklausel nach einem Volksbegehren eingeführt. 
Die praktische Nutzung der Übernahmeklausel ist inzwischen durchaus groß. Zehn Fälle gibt es bislang; der Schwerpunkt lag in den Jahren 2001 bis 2009, und allein vier Fälle geschahen in Hamburg.

(1) Als erster ${ }^{12}$, großer Präzedenzfall gilt der Proteststurm 1978 in Nordrhein-Westfalen gegen eine Schulreform („Kooperative Schule“), als sich beim Volksbegehren 29,8 Prozent der Stimmberechtigten eintrugen (20 Prozent waren erforderlich). ${ }^{13}$ Daraufhin beschloss die Landesregierung, es nicht mehr zum Volksentscheid kommen zu lassen, und gab die umstrittene Reform auf; „das Volksbegehren wurde als Vorwegnahme des Volksentscheids gewertet" ${ }^{14}$. Gemäß der Empfehlung der Landesregierung ${ }^{15}$ beschloss der Landtag einstimmig den volksbegehrten Gesetzentwurf und entsprach damit gemäß Art. 68 Abs. 2 Satz 3 der Verfassung dem Volksbegehren. ${ }^{16}$

(2) 2001 nahm der Niedersächsische Landtag einstimmig den Entwurf eines Kindertagesstättengesetzes an, der sich beim Volksbegehren qualifiziert (11,6 Prozent Eintragungen, nötig waren zehn Prozent) und vor dem Niedersächsischen Staatsgerichtshof mit Maßgaben standgehalten hatte; die kleinen parlamentarischen Änderungen bewegten sich noch innerhalb des Spielraums des Art. 49 Abs. 1 Satz 1 der Verfassung (,im wesentlichen unverändert“). ${ }^{17}$

(3) 2004 reagierte die Bürgerschaft in Hamburg auf das Zustandekommen eines Volksbegehrens für die Kita-Reform (13,9 Prozent ${ }^{18}$ Eintragungen, erforderlich waren fünf Prozent) mit der einstimmigen Verabschiedung eines Gesetzes zur Neuregelung der Hamburger Kinderbetreuung. ${ }^{19}$ Aufgrund des Gestaltungsspielraums des Art. 50 Abs. 3 Satz 1 a.F. der

12 Im Fall „Rundfunkfreiheit“ 1972/73 in Bayern, der in der Literatur mitunter als erster gerechnet wird, kann man zwar einen sachlichen Erfolg des Anliegens des Volksbegehrens feststellen, aber keine formelle Übernahme. Die Landtagsmehrheit lehnte den volksbegehrten Gesetzentwurf ausdrücklich ab, fand sich aber zu einem sehr weitgehenden Entgegenkommen an die Initiatoren bereit, das auf dem regulären Wege zum künftigen Art. 111a der Bayerischen Verfassung führte, vgl. Wolfgang Berger, Die unmittelbare Teilnahme des Volkes an staatlichen Entscheidungen durch Volksbegehren und Volksentscheid, juristische Dissertation, Freiburg im Breisgau 1978, S. 129 -140 .

13 Vgl. Otmar Jung, Daten zu Volksentscheiden in Deutschland auf Landesebene (1946-1992), in: ZParl, 24. Jg. (1993), H. 1, S. 5 - 13, S. 9. Siehe zum Ganzen Werner Blumenthal, Die bildungspolitische Auseinandersetzung und das Volksbegehren um die kooperative Schule in NordrheinWestfalen. Eine Analyse ihrer politischen Faktoren, Ursachen und Strategien sowie der Folgen für die Bildungspolitik in Nordrhein-Westfalen, philosophische Dissertation, Bonn 1988.

14 Andreas Kost, Direkte Demokratie an Rhein und Ruhr, in: Hermann K. Heußner / Otmar Jung (Hrsg.), Mehr direkte Demokratie wagen. Volksentscheid und Bürgerentscheid: Geschichte Praxis - Vorschläge, München 2009, S. 257 - 270, S. 266.

15 Vgl. LT Nordrhein-Westfalen (NRW), Drs. 8/3150 vom 4. April 1978, Anlage 2.

16 Vgl. LT NRW, PIPr Nr. 8/72 vom 13. April 1978, S. 5129 B.

17 Vgl. Niedersächsischer LT, Drs. 14/2832 vom 31. Oktober 2011; PlPr Nr. 14/91 vom 12. Dezember 2001, S. 8885.

18 Nach Angabe der Initiatoren. In Hamburg prüft man die Unterschriften nur noch insoweit amtlich, als es für die Entscheidung über das Zustandekommen des Volksbegehrens erforderlich ist; dann wird die Prüfung abgebrochen. Kritisch dazu Otmar Jung, Direkte Demokratie und bürokratische Anforderungen für Engagierte und öffentliche Verwaltungen, in: AWV - Arbeitsgemeinschaft für wirtschaftliche Verwaltung e.V. (Hrsg.), Bürokratieentlastung des Dritten Sektors und des bürgerschaftlichen Engagements. Notwendigkeit, Praxis und Perspektiven, Eschborn 2011, S. $103-117$, S. $111 \mathrm{ff}$.

19 Vgl. Hamburgische Bürgerschaft (HmbBü), Drs. 18/88 vom 20. April 2004; PlPr Nr. 18/3 vom 21. April 2004, S. 89 AB. 
Verfassung („ein dem Anliegen des Volksbegehrens entsprechendes Gesetz“) entfiel damit der Volksentscheid.

(4) Im Sommer desselben Jahres kam in der Hansestadt ein weiteres Volksbegehren, „Unser Wasser Hamburg“, zustande, wonach „Hamburgs öffentliche Wasserversorgung weiterhin vollständig Eigentum und unter uneingeschränkter Verfügung der Freien und Hansestadt Hamburg" bleiben sollte (12,2 Prozent Eintragungen, fünf Prozent waren nötig). Die Bürgerschaft beschloss einstimmig entsprechend. ${ }^{20}$

(5) Parallel hatte ein zweites Volksbegehren in Hamburg die Hürde überwunden (10,2 Prozent Eintragungen, erforderlich waren fünf Prozent), das sich gegen die Privatisierung der Berufsschulen wandte („Bildung ist keine Ware“). Die Bürgerschaft beschloss mit Mehrheit entsprechend. ${ }^{21}$

(6) 2006 übernahm die Bremische Bürgerschaft mit Mehrheit gemäß Art. 70 Abs. 1 Satz 4 der Verfassung formell einen Wahlgesetzentwurf aus einem erfolgreichen Volksbegehren (13,5 Prozent Eintragungen, erforderlich waren zehn Prozent). ${ }^{22}$

(7) Ein dem Anliegen eines Volksbegehrens (8,2 Prozent Eintragungen, fünf Prozent waren erforderlich) entsprechendes Wahlgesetz verabschiedete 2007 die Hamburgische Bürgerschaft einstimmig und nutzte dabei wieder den verfassungsrechtlichen Gestaltungsspielraum. ${ }^{23}$

(8) 2008 trugen sich 12,0 Prozent der Thüringer Bürgerinnen und Bürger beim Volksbegehren „Mehr Demokratie in Thüringer Kommunen“ ein (zehn Prozent waren erforderlich). Der Landtag übernahm 2009 mit Mehrheit den begehrten Gesetzentwurf. ${ }^{24}$

(9) Im Februar 2013 folgte die eingangs skizzierte Übernahme des Volksbegehrens „Nachtflugverbot“ in Brandenburg, und

(10) nach einem Volksbegehren in Bayern gegen die „Studiengebühren“ im Januar 2013 (14,3 Prozent Eintragungen, zehn Prozent waren erforderlich) übernahm der Bayerische Landtag im April 2013 den begehrten Gesetzentwurf mit übergroßer Mehrheit.

Nun kann dieser Beitrag jene zehn Fälle schon aus Platzgründen nicht vergleichend untersuchen. Er konzentriert sich auf den brandenburgischen Fall, blickt aber, wenn angezeigt, vor allem auf den großen Präzedenzfall in Nordrhein-Westfalen 1978.

20 Vgl. HmbBü, Drs. 18/1223 vom 24. November 2004; PIPr Nr. 18/17 vom 24. November 2004, S. 814 C.

21 Vgl. HmbBü, Drs. 18/1282 vom 24. November 2004; PIPr Nr. 18/17 vom 24. November 2004, S. 812 A. Durch Urteil vom 30. November 2005 erklärte das Hamburgische Verfassungsgericht, dass das verabschiedete Gesetz dem Anliegen des Volksbegehrens entsprochen habe.

22 Vgl. Bremische Bürgerschaft, Drs. 16/1242 vom 12. Dezember 2006, PIPr Nr. 16/72 vom 13. Dezember 2006, S. 4822 C.

23 Vgl. HmbBü, Drs. 18/6341 vom 31. Mai 2007, vor allem S. 7; Drs. 18/6351 vom 4. Juni 2007, S. 4; PIPr Nr. 18/81 vom 6. Juni 2007, S. 4324 C. Hingegen war das 2009 mit 6,2 Prozent Eintragungen (erforderlich waren fünf Prozent) zustande gekommene Volksbegehren „Mehr Demokratie - Ein faires Wahlrecht für Hamburg“ eine Erfolgsgeschichte, aber kein Übernahmefall. Die Vertrauenspersonen verständigten sich mit den in der Bürgerschaft vertretenen Parteien auf einen Kompromiss-Gesetzentwurf, den das Parlament einstimmig verabschiedete (Drs. 19/3255 vom 10. Juni 2009, PIPr Nr. 19/31 vom 24. Juni 2009, S. 1929). Daraufhin zog die Initiative ihren vorsorglich eingereichten Antrag auf Durchführung eines Volksentscheids zurück. Vgl. zum Ganzen Klaus David, Wechselfälle des Wahlrechts in Hamburg. Oder: Die Verhinderung eines weiteren Volksentscheids, in: ZParl, 41. Jg. (2010), H. 3, S. 598 - 622.

24 Vgl. Thüringer LT, Drs. 4/4550 vom 23. Oktober 2008, PIPr Nr. 4/105 vom 3. April 2009, S. 10570. 


\subsection{Einschätzung und Problematisierung}

Die Übernahmeklausel wird im Allgemeinen als positiv eingeschätzt. ${ }^{25}$ Sie ermögliche dem Parlament, responsiv zu handeln, und trage zu einer gewissen Flexibilisierung der Volksgesetzgebung bei; dass der Volksentscheid nach einer Übernahme entfalle, entspreche „reiner Verfahrensökonomie“. ${ }^{26}$ Diese Klauseln haben denn auch die Auslegungskunst der Verfassungskommentatoren nicht angeregt; die Kommentar- und sonstige Literatur ist unergiebig. ${ }^{27}$ Man geht offenbar einfach davon aus, dass das Parlament kraft besserer Einsicht einlenken würde oder sich dem offenkundig entgegenstehenden Volkswillen beugen wolle und insofern bei seiner Entschließung frei sei. Dass das Ermessen des Parlaments insoweit Einschränkungen aus der Logik (direkt-)demokratischer Verfahren unterliegen könnte, erscheint sozusagen undenkbar.

Auch die Spezialliteratur zur direkten Demokratie sah lange keine Probleme. Noch 1994 ging Hermann K. Heußner in seiner großen Studie über die Volksgesetzgebung auf das Stichwort „Chancengleichheit“ aus der älteren Literatur überhaupt nicht ein. ${ }^{28}$ Ein Jahr später machte Stefan Przygode gegen die Übernahmemöglichkeit „gravierende Bedenken“ geltend: Er arbeitete klar den konzeptionellen Fehler dahinter heraus („Unzulässige Isolierung des Volksbegehrens") und nahm unter den Leitfragen der Gleichbehandlung und Chancengleichheit bewusst auch einmal die „Gegnerperspektive“ ein. ${ }^{29} \mathrm{Zu}$ dieser noch ganz abstrak-

25 Damit geht allerdings die Negativ-Wahrnehmung des Volksentscheids als eines Übels einher, das man auf diese Weise - Sprache ist verräterisch - „vermeiden“, „verhindern“, „abwenden“ und dergleichen mehr könne.

26 Vgl. Otmar Jung, Welche Regeln empfehlen sich bei der Einführung von Volksbegehren und Volksentscheid (Volksgesetzgebung) auf Bundesebene?, in: Direkte Demokratie in Deutschland. Handreichungen zur Verfassungsdiskussion in Bund und Ländern. Mit Entwürfen zur Einführung von Volksbegehren und Volksentscheid auf Bundesebene („Hofgeismarer Entwurf"), hrsg. von der Evangelischen Akademie Hofgeismar / Stiftung Mitarbeit, Bonn 1991 (Brennpunkt-Dokumentation Nr. 12), S. 19 - 59, S. 40 f. In diesem Sinne auch Bärbel Martina Weixner, Direkte Demokratie in den Bundesländern. Verfassungsrechtlicher und empirischer Befund aus politikwissenschaftlicher Sicht (Forschung Politikwissenschaft, Bd. 162), Opladen 2002, S. 154: So lassen „sich personelle, zeitliche und finanzielle Ressourcen sparen".

27 Siehe nur Alexander von Brünneck / F. Immanuel Epting, \$22: Politische Gestaltungsrechte und Volksabstimmungen, in: Helmut Simon / Dietrich Franke / Michael Sachs (Hrsg.), Handbuch der Verfassung des Landes Brandenburg, Stuttgart u.a. 1994, S. 339 - 353, S. 350, Rdnr. 16; Hasso Lieber, Art. 78 Nr. 2, S. 510, in: ders. / Steffen Johann Iwers / Martina Ernst, Verfassung des Landes Brandenburg. Kommentar, Wiesbaden 2012; Julia Platter, Volksinitiative, Volksbegehren und Volksentscheid zu Gegenständen der politischen Willensbildung (Art. 76-78 LV), in: Gunter Fritsch (Hrsg.), 20 Jahre Landesverfassung. Festschrift des Landtages Brandenburg, Berlin 2012, S. 113 - 133, S. 116. Von den Belegen für 19 weitere, neuere (seit 2000) Kommentare zu den anderen Landesverfassungen, die ebenfalls konsultiert wurden, wird aus Platzgründen abgesehen. Jedenfalls fanden die Tatbestandsmodifikationen wie ,im wesentlichen unverändert“ Interesse, die ja in den hamburgischen Fällen eine große Rolle spielten. Aber die hier aufgeworfene Problematik wird in dieser Literatur schlichtweg nicht gesehen.

$28 \mathrm{Vgl}$. Hermann K. Heußner, Volksgesetzgebung in den USA und in Deutschland. Ein Vergleich der Normen, Funktionen, Probleme und Erfahrungen (Erlanger Juristische Abhandlungen, Bd. 43), Köln u.a. 1994, S. 296, Fn. 557.

29 Vgl. Stefan Przygode, Die deutsche Rechtsprechung zur unmittelbaren Demokratie. Ein Beitrag zur Praxis der Sachentscheide in Deutschland (Fundamenta Juridica, Bd. 28), Baden-Baden 1995 , S. 160 ff., S. 169. Przygode wurde von der oben erwähnten Kommentarliteratur insoweit nicht rezipiert. 
ten Problementfaltung steuerte Johannes Rux 2008 einen hypothetischen Fall bei, „daß der Gesetzgeber vor dem vermeintlichen Volkswillen, einknickt', obwohl das betreffende Gesetz möglicherweise überhaupt nicht von einer hinreichenden Mehrheit der Bürger unterstützt wird“30. Nun liegt ein solcher Fall konkret vor.

\section{Besonderheiten des Brandenburger Falles}

Zunächst seien einige Grundtatsachen der direkten Demokratie in Erinnerung gerufen: Bei dem Volksbegehren in Brandenburg handelte es sich nur um einen Relevanztest, ob das Anliegen „Nachtflugverbot“ dem Volk zur Entscheidung vorgelegt werden sollte. Erforderlich war dafür bloß die Eintragung von umgerechnet 3,8 Prozent der Stimmberechtigten; es reichte also die Willensbekundung einer kleinen Minderzahl aus, um das Verfahren weiterzutreiben. Dieser Test wurde mit der Eintragung von fünf Prozent bestanden. Ein Volksbegehren ist aber nicht die Entscheidung des Volkes selbst. Ob am Ende des Verfahrens bei einer Volksabstimmung eine Mehrheit an den Urnen für jenes Anliegen votieren wird, ist offen; erst recht, ob gegebenenfalls diese Mehrheit der Abstimmenden das Quorum von 25 Prozent Zustimmung der Stimmberechtigten nach Art. 78 Abs. 2 BbgVerf erfüllt - das würde immerhin rund 530.000 Ja-Stimmen erfordern gegenüber jetzt 106.000 geleisteten Unterschriften beim Volksbegehren. ${ }^{31}$

Keinesfalls ähnelt die Situation nach dem Zustandekommen dieses brandenburgischen Volksbegehrens dem Präzedenzfall in Nordrhein-Westfalen 1978:

Erstens macht es einen gewaltigen Unterschied, ob fast ein Drittel (wie 1978 in Nordrhein-Westfalen) oder gerade einmal ein Zwanzigstel der Stimmberechtigten (wie 2012 in Brandenburg) ein Volksbegehren unterstützen. Brandenburg hat die niedrigste Volksbegehrenshürde im Ländervergleich (80.000 Eintragungen ${ }^{32}$ ). Dies ist vom Standpunkt der direkten Demokratie aus erfreulich. Als Kehrseite beeindruckt es allerdings wenig, wenn eine so niedrige Hürde genommen wird. ${ }^{33}$ Von einer Vorwegnahme des Volksentscheids kann jedenfalls hier keine Rede sein.

Zweitens zeigt die Verteilung der Eintragungen auf die kreisfreien Städte und Landkreise des ganzen Landes, dass das Anliegen „Nachtflugverbot“ zwar in der Flughafenregion Reso-

30 Vgl. Johannes Rux, Direkte Demokratie in Deutschland. Rechtsgrundlagen und Rechtswirklichkeit der unmittelbaren Demokratie in der Bundesrepublik Deutschland und ihren Ländern, BadenBaden 2008, S. 319, Fn. 6.

31 Von daher ist der Behauptung des Landesverbandes Berlin-Brandenburg von Mehr Demokratie e.V. zu widersprechen, „das Ergebnis des Volksbegehrens komme einem klaren Auftrag für die Landesregierung gleich" (Michael Schlieben, Wie Platzeck die Nachtruhe entdeckte, in: Zeit online vom 22. Februar 2013, http://www.zeit.de/politik/deutschland/2013-02/brandenburg-platzeck-flughafen (Abruf am 23. Februar 2013)). Dies wäre erst nach einem Sieg beim Volksentscheid der Fall.

32 Art. 77 Abs. 3 Satz 1 BbgVerf. Irrig daher Stephan-Andreas Casdorff, Erklär mir den Platzeck, in: Der Tagesspiegel vom 4. März 2013: „Die Hürde ist hoch.“

33 Gewiss kann man dieses einzige Volksbegehren, das bisher in Brandenburg zustande kam, „das größte und erfolgreichste in der Geschichte" des Landes nennen (so Michael Schlieben, a.a.O. (Fn. 31)). Aber über dieser Binnenperspektive sollte man nicht die Maßstäbe außer acht lassen, die der Ländervergleich bietet. Die Folgerung des Autors: „Daher lässt sich die Kehrtwende auch unter demokratietheoretischen Aspekten nachvollziehen“, ist überzogen. 
nanz, jedoch „in den Weiten der Mark kaum Unterstützer fand“34. Man könnte realistischerweise eher von einem Betroffenenbegehren als von einem Volksbegehren sprechen. ${ }^{35}$

Drittens sollen nach einer Forsa-Umfrage von Ende Dezember 2012 sich landesweit 54 Prozent der Befragten gegen ein strengeres Nachtflugverbot am neuen Flughafen und nur 43 Prozent für das Anliegen des Volksbegehrens ausgesprochen haben. ${ }^{36}$ Kurz vor der Landtagsentscheidung tauchten neuere demoskopische Daten auf. Nach einer Emnid-Umfrage im Auftrag der Linken sollen sich 48 Prozent der Brandenburger gegen und weiterhin nur 43 Prozent für ein strengeres Nachtflugverbot ausgesprochen haben. Eine dritte Umfrage, welche die Landes-SPD in Auftrag gegeben hatte und deren Ergebnisse sie unter Verschluss hielt, soll ähnlich ausgefallen sein. Jedenfalls blieb ein Vorsprung derer, die das Anliegen des Volksbegehrens ablehnten, erhalten. ${ }^{37}$

Im Vergleich zeigen sich erhebliche Unterschiede zum Präzedenzfall 1978 in NordrheinWestfalen: Statt einer starken bloß eine schwache Unterstützung beim Volksbegehren, statt eines landesweit getragenen Protestes nur ein regional begrenzter, und statt eines klar voraussagbaren Ausgangs des Volksentscheids damals herrschte jetzt - so viel gab die SPD aus ihrer Umfrage preis - „eine Fifty-Fifty-Stimmung“"38. Was bedeutete es, in dieser Situation das direktdemokratische Verfahren durch den Übernahmebeschluss des Landtags zu kupieren?

\section{Direkte Demokratie: Befriedung bei Fairness}

Die befriedende Wirkung der direkten Demokratie beruht darauf, dass alle aufgerufen sind, sich an der Beratung einer konkreten Sachfrage zu beteiligen, und dass alle die verbindliche Antwort auf diese Frage durch ihre Stimmabgabe beeinflussen können. Dieser überaus positi-

34 Klaus Kurpjuweit / Thorsten Metzner, Politische Luftnummer, in: Der Tagesspiegel vom 5. Dezember 2012.

35 Dies ist nicht abwertend gemeint. Selbstverständlich haben auch Betroffene das Recht, sich der Instrumente der direkten Demokratie zu bedienen, ja ihre Betroffenheit wird sie zu allererst zu ihrem Engagement motivieren. Es soll nur ausdrücken, dass das hohe Interesse der Lärmgeplagten mit einem niedrigen allgemein-staatsbürgerlichen Interesse der Brandenburger außerhalb der betroffenen Gebiete kontrastierte.

36 Die Umfrage hatte die „Märkische Allgemeine Zeitung“ in Auftrag gegeben, vgl. Torsten Gellner, Mehrheit lehnt lange Nachtflugpause ab. MAZ-Umfrage: 75 Prozent glauben nicht an BER-Eröffnungstermin, 28. Dezember 2012, http://www.maerkischeallgemeine.de/cms/ziel/604050/ DE?article_id=2729467 (Abruf am 22. Februar 2013).

37 Dass „die Stimmung im Land deutlich in Richtung eines Nachtflugverbots gekippt“ wäre, ist nach den letzten Zahlen ein falsches Bild. Besser träfe, dass der Vorsprung derer, die das Volksbegehren ablehnten, geschmolzen sei. Dass Platzeck daher „bei einem Volksentscheid im Sommer womöglich eine Niederlage gedroht hätte" - also eine Ja-Mehrheit zu dem Anliegen des Volksbegehrens -, wirkt nur unter der ziemlich spekulativen weiteren Annahme plausibel, dass ein Volksentscheid „vor allem Nachtflug-Gegner und BER-Kritiker mobilisiert“ hätte (alle Zitate bei Thorsten Metzner, Hinterhergedüst, in: Der Tagesspiegel vom 27. Februar 2013, S. 12). Als Erklärung für Platzecks Kehrtwende reichen diese Daten kaum aus. Im Übrigen ist die letzte Frage für diesen Beitrag gar nicht so wichtig. Es geht darum, was der Übernahmebeschluss des Landtags objektiv bewirkte, nicht darum, was Platzeck subjektiv dazu bewog, diesen Beschluss in die Wege zu leiten.

38 Ders., Platzeck hätte bei Volksentscheid Niederlage gedroht, in: Potsdamer Neueste Nachrichten vom 26. Februar 2013, http://www.pnn.de/brandenburg-berlin/728116/ (Abruf am 27. Februar 2013). Diese Information aus dem Schwesterblatt ist im gleichen Artikel des Autors im „Tagesspiegel“ (Fn. 37) nicht enthalten. 
ve Effekt kann allerdings (noch) nicht auf der ersten Verfahrensstufe des Volksbegehrens eintreten (geschweige denn bei der mitunter vorgeschalteten Volksinitiative). Hier steht die Initiativfunktion im Vordergrund, der Relevanztest muss bestanden werden, und in den allermeisten Fällen ${ }^{39}$ engagieren sich Befürworter des im Volksbegehren formulierten Anliegens. Bloß eine Seite fordert also, argumentiert, wirbt. Für die andere Seite - man denke an die "Gegenakteure " 40 und deren Basis - gibt es in dieser Phase kaum eine strukturierte Möglichkeit der Artikulation. Anders bei der öffentlichen Abstimmungsdebatte vor der zweiten Verfahrensstufe des Volksentscheids. Nun bilden sich aus den Befürwortern eines Anliegens und den Sachgegnern typischerweise „Volksentscheidsparteien“; beide Seiten geben Abstimmungsempfehlungen aus, argumentieren und werben dafür. Diese öffentliche Auseinandersetzung ist äußerst wichtig ${ }^{41}$, und sie muss diszipliniert, ja kultiviert geführt werden als Streit zwischen demokratischen Bürgern um die beste Sachlösung. Zu den Essentialia demokratischer Kultur gehört dabei zumindest, dass man der jeweils anderen Seite den guten Willen nicht abspricht und dass man bereit ist, den Entscheid der Mehrheit an den Urnen praktisch zu akzeptieren.

Dieses Modell der direkten Demokratie wird gestört, wenn bei einem hochkontroversen Gegenstand das Parlament bereits nach dem Volksbegehren das Anliegen der Initiatoren übernimmt. Das ganze Verfahren wirkt nun unfair: Der Freude der lärmbetroffenen Unterzeichner des Volksbegehrens über ihren raschen Sacherfolg steht das ärgerliche Gefühl derer, die - vereinfacht gesagt - die ökonomischen Aspekte dieses Flughafens höher bewerten, gegenüber, dass sie überhaupt „nicht gefragt“ wurden. ${ }^{42}$ Dies ist in der Tat ein irritierender Befund: Die Position der Andersdenkenden, die es doch ausweislich der Umfragen zu Hunderttausenden gab, wurde sowohl sachlich als auch hinsichtlich ihrer fairen Artikulationsmöglichkeiten völlig ausgeblendet - von den Protagonisten, von den Debattenrednern im Landtag ${ }^{43}$ und von der politischen Öffentlichkeit. ${ }^{44}$ Beim Landtag war dies besonders misslich. Für die Übernah-

39 Theoretisch können sich natürlich auch Bürger beim Volksbegehren eintragen, die aus prinzipiellen Erwägungen wollen, dass diese Causa nicht repräsentativ-demokratisch vom Parlament, sondern direktdemokratisch vom Volk entschieden wird, aber sich vorbehalten, bei der abschließenden Sachabstimmung mit „Nein“ zu votieren. Doch dies dürfte eher selten vorkommen.

40 Vgl. zu diesen Theo Schiller, Direkte Demokratie. Eine Einführung, Frankfurt am Main 2002, S. 150 .

41 Vgl. ebenda, S. 146: „Für den Demokratieanspruch direktdemokratischer Verfahren ist diese öffentliche Debatte jedenfalls von entscheidender Bedeutung."

42 Stefan Przygode, a.a.O. (Fn. 29), S. 162, pointierte: „Nur wenn der ordentliche Gesetzgeber den Gesetzesvorschlag ablehnt, haben die Gegner überhaupt die Chance, im laufenden Verfahren ihre Meinung in rechtlich erheblicher Weise zur Geltung zu bringen."

43 Die Parlamentsdebatte am 27. Februar folgte weitgehend dem Muster einer parteipolitischen Auseinandersetzung. Allein der Abgeordnete Gregor Beyer (FDP) kam zumindest in die Nähe des Problems, als er die Forderungen der Lärmbetroffenen unverblümt „Partikularinteressen“ nannte und betonte, „dass die Entscheidung darüber ausschließlich in die Hand des Souveräns gehör(e)“, LT Bbg, PlPr Nr. 5/71 vom 27. Februar 2013, S. 5766 f.

44 So begrüßte der Landesverband Berlin-Brandenburg von Mehr Demokratie e.V., obwohl satzungsgemäß sachpolitisch neutral und allein der Förderung der direkten Demokratie verpflichtet, sogleich die Übernahme des Anliegens des Volksbegehrens „Nachtflugverbot“ durch die Landesregierung. Dies zeige, dass die rot-rote Koalition „die Unterschriften von 106.000 Bürgerinnen und Bürgern ernst" nehme (vgl. PM 6/13 vom 19. Februar 2013). Entsprechend verteidigte seine PM 7/13 vom 26. Februar 2013 die Brandenburger Landesregierung, weil sie auch „Bedenken der Bürgerinnen und Bürger ernstnimmt“. Dass dieselbe Regierung nach den entgegengesetzten Bedenken der Andersdenkenden gar nicht fragte, wurde konsequent übersehen. 
meentscheidung reichte die Regierungsmehrheit; dann hätte das Parlament immer noch als „Tribüne“ des Landesvolkes die öffentliche Auseinandersetzung, die man auf der Ebene der Bürger weggekürzt hatte, stellvertretend führen können. Aber auch diese Chance wurde vergeben, als die Abgeordneten - in Koalition und Opposition -, wie Debatte und Abstimmung zeigten, vor den Initiatoren mehr oder minder „einknickten“. Mehr noch: Wenn die Organe der repräsentativen Demokratie sich so mit den Vertretern einer aktivistischen Minderheit „kurzschließen“, dürften angesichts der erwähnten Umfragen viele argwöhnen, dass sie einfach „überspielt“ wurden. ${ }^{45}$ Solche Gefühle bewirken natürlich das Gegenteil einer Befriedung. ${ }^{46}$ Aber auch die Befürworter zahlen einen Preis: Wenn das Ziel des abschließenden Entscheids des ganzen Volkes aus den Augen gerät, verliert die Verfahrensstufe des Volksbegehrens ihre spezifische Dignität ${ }^{47}$ und sinkt herab zu einer Art Volks-Großinitiative. ${ }^{48}$

Umgekehrt werden die Initiatoren bei der Fortführung des direktdemokratischen Verfahrens nicht unfair belastet. Sie haben gewusst, worauf sie sich einließen. Es war klar, dass das Bestehen des Relevanztests Volksbegehren noch die leichtere Aufgabe war, der die schwerere folgen würde, eine Mehrheit an den Urnen zu erringen. Dass ihnen diese zweite Aufgabe durch die Übernahme erlassen wurde, mögen sie bejubeln. Einen Grund, sich zu beklagen, wenn ihnen dieses politisch zweifelhafte Geschenk nicht gemacht worden wäre, hätten sie nicht gehabt. Was sie verlangen können - und darauf hätte Platzeck seine Energie richten sollen -, ist eine Auseinandersetzung unter fairen Bedingungen. Dazu gehören die Abschaffung der Abstimmungsquoren mit ihren Verzerrungseffekten ${ }^{49}$ und die Einführung einer angemessenen Kostenerstattung für Volksbegehren und Volksentscheid, die bislang in Brandenburg fehlt. ${ }^{50}$

\section{Platzecks Begründungen}

Was Platzeck, die Landesregierung beziehungsweise die Koalitionsredner zur Begründung ihres Umschwenkens vorbrachten, vermag es kaum, den Vorwurf der Unfairness zu entkräften; vielmehr zeugt es von tiefgreifenden Missverständnissen der direkten Demokratie.

45 Die Gefahr einer „Volksgesetzgebung ohne Volk“ sieht bei solchen Konstellationen Ralf Kleindiek, Machen Volksgesetzgebung und Transparenz unsere Demokratie besser?, in: Michael Bäuerle I Philipp Dann / Astrid Wallrabenstein (Hrsg.), Demokratie-Perspektiven. Festschrift für Brun-Otto Bryde zum 70. Geburtstag, Tübingen 2013, S. 175 - 198, S. 194.

46 Vgl. Stefan Przygode, a.a.O. (Fn. 29), S. 160 f.: „Das dem Volksgesetzgebungsverfahren immanente Selbstregulierungsmoment, die eigene Entscheidung aus dem und durch das Volk in eigenen Angelegenheiten, welche zudem mit einer hohen Akzeptanz einhergeht sowie mit einem hohen Befriedungsmoment verbunden ist, wird durch diese unzutreffende Teilsichtweise zunichte gemacht."

47 Vgl. ebenda, S. 160: „Diese isolierte Sicht ignoriert gänzlich die Eigenständigkeit und Einheitlichkeit des Volksgesetzgebungsverfahrens, mithin Sinn und Zweck des Verfahrens insgesamt. (...) Das Volksbegehren ist ein Antrag aus dem Volk an das Volk und hat einen Volksentscheid zum Ziel.“

48 Statt von mindestens 20.000 Einwohnern (Art. 76 Abs. 1 Satz 3 BbgVerf) nun eben von 80.000 Stimmberechtigten (Art. 77 Abs. 3 Satz 1 BbgVerf) unterstützt.

49 Vgl. Frank Meerkamp, Die Quorenfrage im Volksgesetzgebungsverfahren. Bedeutung und Entwicklung (Bürgergesellschaft und Demokratie, Bd. 36), Wiesbaden 2011, S. 469, S. 478.

50 Vgl. Gunther Jürgens / Frank Rehmet, Direkte Demokratie in den Bundesländern - Ein Überblick, in: Hermann K. Heußner / Otmar Jung (Hrsg.), a.a.O. (Fn. 14), S. 197 - 233, S. 208. 


\subsection{Spaltung}

Als Hauptgrund wurde die Gefahr der Spaltung genannt. Zu der befürchteten Vertiefung der Spaltung könnte es durchaus kommen, wenn jede Seite, um mit Platzeck zu sprechen, mit einer monatelangen „Kampagne“ polarisieren würde, womöglich mit einer „Schlammschlacht" wie in der so genannten heißen Wahlkampfphase. Aber müsste das so kommen, beziehungsweise trüge, wenn es denn so wäre, nicht die Regierung ein Gutteil der Verantwortung? Dass es auch anders geht, hat Ende 2011 die Volksabstimmung in Baden-Württemberg über das Bahnprojekt „Stuttgart 21“ bewiesen. ${ }^{51}$ Das war kein Zufall. Allein die Tatsache, dass jetzt sowohl die Befürworter eines Vorhabens als auch die Gegner aktiv teilnehmen können, „macht den Abstimmungskampf zu einem konstruktiven sachpolitischen Streit über den politisch einzuschlagenden Weg"52. Vertieft es nicht gerade umgekehrt die Spaltung, wenn die Regierung dem artikulierten Willen von 106.000 Bürgern umgehend entspricht, aber Hunderttausenden anderen Bürgern gar keine Möglichkeit der Artikulation ihres laut Umfragen entgegenstehenden Willens mehr gibt? Eine monatelange gute Diskussion kann statt zu einer Zuspitzung der Gegensätze, wie sie Platzeck als zwangsläufig sieht ${ }^{53}$, zu einem besseren Verständnis der verschiedenen Pro- und Contra-Argumente und damit zu einer Entschärfung führen ${ }^{54}$ - auch wenn am Ende natürlich eine Entscheidung steht, die die eine Seite als „Sieg“ und die andere als „Niederlage“ wahrnehmen wird. ${ }^{55}$ Dabei kann eine Regierung noch Gutes beisteuern, indem sie alles tut für eine möglichst hohe Beteiligung an der Abstimmung, um ein „unechtes Scheitern“ am Zustimmungsquorum zu ver-

51 Vgl. Thorsten Faas / Johannes N. Blumenberg, Jenseits der Volksabstimmung: Einstellungen zu „Stuttgart 21" und zur Demokratie in Baden-Württemberg, 2010-2012, in: Frank Brettschneider I Wolfgang Schuster (Hrsg.), Stuttgart 21. Ein Großprojekt zwischen Protest und Akzeptanz, Wiesbaden 2013, S. 299 - 315, S. 308, S. 315. Dies lag allerdings auch an dem (nach direktdemokratischen Kriterien) „guten“ Ausfall der Volksabstimmung, vgl. Otmar Jung, Stuttgart 21 und die Direkte Demokratie. Kritische Anmerkungen zum Umgang mit dem Ergebnis der Volksabstimmung, in: Recht und Politik, 48. Jg. (2012), H. 1, S. 11 - 17, S. 12 ff.: Es wurde eine klare Entscheidung getroffen, ohne Verzerrung durch Quoren, und es fand sich sogar bei den unmittelbar Betroffenen im Stadtkreis Stuttgart eine Mehrheit, die mit der Landesmehrheit gleichlief.

52 Peter Neumann, Sachunmittelbare Demokratie im Bundes- und Landesverfassungsrecht unter besonderer Berücksichtigung der neuen Länder, Baden-Baden 2009, S. 814 (Rdnr. 2173).

53 Vgl. Ministerpräsident Platzeck: „Im Lande wird es danach ganz automatisch keine flacheren Gräben geben, sondern sie werden nach einer solchen Kampagne eher noch tiefer sein. Auch das ist doch normal." LT Bbg, PIPr Nr. 5/71 vom 27. Februar 2013, S. 5770. Zu diesem Argument hatte bereits vorher der FDP-Abgeordnete Gregor Beyer geätzt: „Ist es schon so weit gekommen, dass Debatten im Rahmen eines Volksentscheids schädlich sind?“" (LT Bbg, PIPr Nr. 5/71 vom 27. Februar 2013, S. 5766).

54 Siehe dazu Monika Böhm, Die direkte Demokratie in der Schweiz - Ein Vorbild für Deutschland?, in: DÖV, 66. Jg. (2013), S. 1 - 7, S. 7: „Die breiten öffentlichen Diskussionen, ein diskursives Politikverständnis und eine offenere Meinungsvielfalt auch innerhalb der politischen Parteien führen zu einer entspannteren Diskussion, als dies bei einer stärkeren Monopolisierung der Entscheidungsfindung in den Parlamenten der Fall ist" (Hervorhebung nicht im Original).

55 Theo Schiller, a.a.O. (Fn. 40), hat denn auch betont, die intensive öffentliche Debatte, die breite Beteiligungsmöglichkeit daran und das Entscheidungsrecht aller trügen ,zur politischen Integration der Bürgerinnen und Bürger in die politische Gemeinschaft“ bei, „, unabhängig davon, wer im Einzelfall bei einer Entscheidung erfolgreich war" (S. 163). Die von Platzeck angeführte Gefahr einer Vertiefung der Spaltung des Landes könnte man mit Schiller zu den „fast klischeehafte(n) Negativannahmen" rechnen, die es zu öffentlichen Abstimmungsdebatten gibt (S. 41). 
hindern, das immer Unzufriedenheit nährt. Eine klare Entscheidung mit Ja- oder NeinMehrheit indes hat eine befriedende Wirkung, was sich in Berlin zuletzt bei der Ablehnung des „Pro-Reli“-Vorstoßes an den Urnen gezeigt hat. ${ }^{56}$ Doch statt diese große DemokratieÜbung zuzulassen, sie auch „auszuhalten“ und möglichst gut zu gestalten, hat Platzeck in einer Art paternalistischem Reflex die Angelegenheit den Bürgern wieder weggenommen und für die repräsentative Demokratie beansprucht. Man ist versucht zu spotten: Wenn es das erste Mal ernst wird, verhält sich der erklärte Freund politischer Partizipation wie ein Vater, der die Entscheidung an sich zieht, weil die Kinder sich ja doch nicht vernünftig auseinandersetzen, sondern sich nur „,in die Haare geraten“ würden.

\subsection{Zeitgewinn}

Als zweiter Grund wurde genannt, dass man den Zeitverlust durch das weitere Verfahren bis zum Volksentscheid vermeiden wolle. ${ }^{57}$ Zweifelsohne kostet ein Volksentscheid Zeit. Aber der Verfassungsgeber hat die direkte Demokratie in Brandenburg bewusst als ein langsames Verfahren ausgestaltet, das jeder Überstürzung vorbeugt und hinreichend Zeit lässt, um reflektierend und abwägend einen Volkswillen zu bilden. Gerade in diesem Sinne hatte Brandenburg (unter Platzecks politischer Führung) noch im Dezember 2011 die Eintragungsfrist beim Volksbegehren von vier auf sechs Monate verlängert. ${ }^{58}$ Umso mehr verwundert es, dass nun die vier Monate bis zu einem Volksentscheid - als Tag der Abstimmung hatte man bereits den 16. Juni ins Auge gefasst ${ }^{59}$ - nicht mehr zur Verfügung stehen sollten, und dass bei einem Flughafen, für dessen Fertigstellung derzeit niemand einen Termin anzusetzen wagt. Bei einem Thema, das selbst mit der Aufnahme des Flugbetriebs nicht erledigt ist - selbstverständlich kann man dann immer noch über ein erweitertes Nachtflugverbot verhandeln. ${ }^{60}$

Vor allem aber wäre diese Zeit mitnichten verloren, sondern sie hätte gerade die spezifischen Gewinne der direkten Demokratie erbringen können. Andreas Gross mit seinen Erfahrungen aus der schweizerischen Praxis betont diesen Aspekt: Kommunikation und öffent-

56 Vgl. Otmar Jung, Direkte Demokratie in Berlin. Der Fall „Pro Reli“ 2007-2009, Berlin 2011, S. $155-163$.

57 Platzeck brachte in einem Interview das Spaltungs- und das Zeitargument noch einmal zusammen: Es gehe „um die Frage: Was tut dem Land gut? Eine monatelange Volksentscheid-Kampagne, die Gräben im Land vertieft und am Ende das gleiche Ergebnis zeitigt wie das Volksbegehren, nämlich die Aufforderung an die Landesregierung, in Verhandlungen mit Berlin einzutreten? Oder ist es besser, möglichst schnell einen Kompromiss zu finden? Ich bin für die Kompromisssuche jetzt.“ André Bochow, Matthias Platzeck: „Ich erwarte am Ende keinen Jubel“, in: Märkische Oderzeitung vom 23. Februar 2013, http://www.moz.de/artikel-ansicht/dg/0/1/1109349/ (Abruf am 23. Februar 2013).

58 Art. 77 Abs. 3 Satz 1 n.F. BbgVerf.

59 Hier gibt es Spielräume: Der Volksentscheid muss zwar innerhalb von drei Monaten nach der Entscheidung des Landtags stattfinden; für diese kann sich das Parlament aber zwei Monate Zeit lassen, vgl. Art. 78 Abs. 1 Satz 1 BbgVerf.

60 Vielleicht wäre dieser Realitätstest sogar nützlich, um entweder den Grund zum Eingreifen mit Messungen nachweisbar zu machen oder aber zum Arrangement mit der Situation. 
liche Deliberation seien gleichsam die „Seelen der Direkten Demokratie“61. Daraus ergebe sich die Forderung nach „mehr öffentlicher Nachdenklichkeit, mehr Diskussion, mehr Gesprächen, mehr gemeinsamer Reflexion, mehr gesellschaftlichen Aushandlungsprozessen, mehr politischer Vermittlung und mehr Ausgleich“62. Indes: „Nachdenken, Diskussion, Begegnungen und Interaktionen benötigen Zeit. Ebenso Aushandlungsprozesse und Verständigungsanstrengungen zwischen Vertretern verschiedener Interessen und Organisationen. Wird diese Zeit nicht gewährt, so bevorteilen die Verfahren wiederum die etablierten Interessen, die ressourcenmächtigen Interessen und jene Kräfte, welche der Auseinandersetzung ohnehin aus dem Wege gehen möchten. Ganz abgesehen davon, dass ohne verfahrensspezifische Einräumung von genügend Zeit der Gewinn an Integration praktisch unmöglich wird.“ Daher dürfe „niemals zu schnell, beispielsweise schon ein halbes Jahr nach Abgabe der Unterschriften, abgestimmt werden"63. Von dieser direktdemokratischen Kultur sind Ministerpräsident Platzeck und seine Mitstreiter offenkundig sehr weit entfernt.

Zudem hatte er den „Zeitgewinn“ um den hohen Preis einer legitimatorischen Schwächung erkauft. Bis dahin war seine machtpolitische Situation relativ bequem gewesen: Hätte eine Mehrheit der Brandenburger an den Urnen mit Nein gestimmt, wäre das Anliegen „Nachtflugverbot“ fair abgelehnt und das Problem damit politisch weitestgehend erledigt gewesen. Hätte sich dagegen eine Mehrheit beim Volksentscheid für ein Nachtflugverbot ausgesprochen (und wäre auch das Zustimmungsquorum erreicht worden), hätte Platzeck mit dem Anspruch, den Volkswillen loyal umzusetzen (Organtreue!) ${ }^{64}$, versuchen können - wie das Volksbegehren formulierte -, „in Verhandlungen mit dem Land Berlin einzutreten“. Schwer genug wäre dies für den Minderheitsgesellschafter - Brandenburg hält 37 Prozent Anteile an der Flughafen-Gesellschaft (ebenso das Land Berlin, die Bundesrepublik Deutschland hält 26 Prozent) - ohnehin geworden.

Durch seine Voreiligkeit provozierte der Regierungschef nun den Vorwurf, er habe auf die Forderung gerade einmal eines Zwanzigstels des Stimmvolkes überreagiert, obwohl die Mehrheit der Brandenburger laut Meinungsumfragen anders denke. Damit schwächte Platzeck seine Verhandlungsposition ${ }^{65}$; nun musste er von Berlins Regierendem Bürgermeister Klaus Wowereit (ebenfalls SPD) verbale Prügel einstecken (von einer „Wutrede“ sprach

61 Andreas Gross, Das Design der Direkten Demokratie und ihre Qualitäten. Erfahrungen und Reformideen im Vergleich zwischen Kalifornien, Schweiz und den deutschen Bundesländern, in: Theo Schiller / Volker Mittendorf(Hrsg.), Direkte Demokratie - Forschungsstand und Perspektiven, Wiesbaden 2002, S. 331 - 339, S. 336.

62 Ebenda, S. 332.

63 Ebenda, S. 337.

64 Diesen Effekt sah die PM 7/13 des Landesverbandes Berlin-Brandenburg von Mehr Demokratie e.V. vom 26. Februar 2013 realistisch. Die Position der Brandenburger Landesregierung hätte durch einen Erfolg beim Volksentscheid „mehr Gewicht“ erhalten, ein solches Ergebnis hätte „für mehr Druck auf die Verhandlungspartner sorgen“ können. Nicht bedacht wurde, dass nach den Umfragen die Mehrheit der abstimmenden Bürger das volksbegehrte Anliegen wahrscheinlich schweizerisch gesprochen - „bachab“ geschickt hätte.

65 Sein Diktum, die Landesregierung habe nach einem Volksentscheid "genau denselben Verhandlungsauftrag“" den sie durch den Landtagsbeschluss, ,jetzt auch“ bekomme (LT Bbg, PIPr Nr. 5/71 vom 27. Februar 2013, S. 5770), ignorierte den potentiellen Gewinn an Legitimation durch eine Volksabstimmung. Zum Ausgleich dieser legitimatorischen Schwäche würden die potentiellen Verhandlungspartner wohl kaum Platzecks Begründung akzeptieren, er wolle „möglichst schnell“ einen Kompromiss finden, vgl. André Bochow, a.a.O. (Fn. 57). 
man gar) und sich in der Presse als „Machiavelli vom Dorfe“ schmähen lassen, der „zunächst märkische Innenpolitik“ betreibe. ${ }^{66}$

\section{Ergebnis: Einschränkungen für die Übernahme}

Das Volksbegehren „Nachtflugverbot“ war von vornherein ein unglückliches Unterfangen. Um die letztlich grundrechtlichen Anliegen der vom Projekt des Flughafen Berlin Brandenburg International Betroffenen geltend zu machen, ist das Planfeststellungsverfahren der richtige Ort. Beim Volkgesetzgebungsverfahren hingegen sind alle Brandenburger zur Entscheidung aufgerufen; es ist nicht dazu da, um Forderungen, die die lärmgeplagten Anwohner beim Abwägungsprozess des Planfeststellungsverfahrens nicht genügend berücksichtigt sehen, sozusagen per Volks-Korrektur doch noch durchzusetzen. Die staatsbürgerliche Selbstregierung der direkten Demokratie ist nicht die „Berufungsinstanz“ für jene, die mit der Betroffenenpartizipation unzufrieden sind.

Wenn man das Volksgesetzgebungsverfahren im Falle „Nachtflugverbot“ aber aus formalen Gründen zuließ und durchführte, sollte man es auch respektieren und auf seine positiven Wirkungen setzen. In Deutschland legen die Regelwerke zwar seit jeher das so genannte indirekte Verfahren der Volksgesetzgebung fest, das auf jeder Verfahrensstufe das Parlament einbezieht. ${ }^{67}$ Von daher ist unter anderem die Möglichkeit folgerichtig, dass das Parlament das Anliegen des Volksbegehrens übernimmt. Indes erscheint es unterkomplex zu meinen, eine solche Übernahme sei schlechterdings erfreulich im Sinne der politischen Kultur. Es kommt vielmehr sehr auf die Umstände an. Mit Blick auf den großen Präzedenzfall in Nordrhein-Westfalen 1978 und jetzt die Vorgänge in Brandenburg sind Einschränkungen nötig.

Positiv formuliert: Eine Übernahme liegt nahe, wenn ein Volksbegehren derart nachdrücklich unterstützt wird, dass man darin die Vorwegnahme des Volksentscheids sehen kann. Das ist keinesfalls gleichbedeutend mit der Forderung nach einer 30-Prozent-Unterstützung. Der Koop-Fall in Nordrhein-Westfalen war ein singulärer Protest; eine Krise der Regierenden und ein zivilgesellschaftlicher „Aufstand“ kamen zusammen, als sich nicht weniger als 3.636.932 Bürger beim Volksbegehren eintrugen. Zu einer maßvollen Bewertung hilft die „Hitliste“ der weiteren Volksbegehren: 18,4 Prozent in Hamburg 1998 (zur Volksgesetzgebung, zehn Prozent waren erforderlich), 18,3 Prozent in Thüringen 2000 (zur Volksgesetzgebung, die Hürde lag bei 14 Prozent) und 18,1 Prozent in Hamburg 1998 (zum bezirklichen Bürgerentscheid, nötig waren zehn Prozent). Die meisten zustande gekomme-

66 Vgl. Thorsten Metzner, Machiavelli vom Dorfe, in: Der Tagesspiegel vom 23. Februar 2013: „Platzecks 180-Grad-Wende ist zunächst märkische Innenpolitik. Um einen Volksentscheid abzuwenden, der zur gefährlichen Abstimmung über das Pannenprojekt geworden wäre, kurz vor der Bundestagswahl, ein Jahr vor der Landtagswahl. Machiavellis Lehren haben Platzeck und seine SPD tief verinnerlicht.“ Der Angesprochene wies dies - natürlich - zurück: „(...) wenn die Frage darauf abzielt, ob ich auf die Wahlen schiele, wenn ich mich um die Nachtruhe in Flughafennähe kümmere: Vergessen Sie es. Bis dahin sind es noch anderthalb Jahre. An die Wahlen denke ich noch lange nicht." André Bochow, a.a.O. (Fn. 57).

$67 \mathrm{Vgl}$. Theo Schiller, a.a.O. (Fn. 40), S. 116, mit Hinweis auf die in den USA häufiger praktizierten direkten Verfahren. Einige Übernahmefälle aus US-Bundesstaaten bringt Hermann K. Heußner, a.a.O. (Fn. 28), S. 306 f. 
nen Volksbegehren liegen - auch dank der zwischenzeitlich erfolgten Hürdensenkungen im einstelligen Bereich. ${ }^{68}$ Eine zahlenmäßige Festlegung, was unter nachdrücklicher Unterstützung zu verstehen ist, braucht hier gar nicht versucht zu werden. Klar ist jedenfalls: fünf Prozent, bei 3,8 geforderten Prozent, sind es nicht. ${ }^{69}$ Man mag dagegen einwenden, dass die Bürger in den Ländern mit niederschwelligen Regelwerken dann in gewisser Weise im Nachteil seien. Doch dies ist unvermeidlich. Man kann nicht beides zusammen haben: die Qualifikationshürde niedrig ansetzen und eine imposante Unterstützung im gerade skizzierten Sinne erwarten, zumal wenn auch andere „Stellschrauben“, etwa die Deckelung der Kostenerstattung auf die erforderliche Zahl der Eintragungen beim Volksbegehren ${ }^{70}$, in die Gegenrichtung wirken.

Negativ formuliert: Eine Übernahme sollte unterbleiben, wenn das direktdemokratische Verfahren unfair zu werden droht. Auch hier ist ein Schwellenwert anzusetzen. Dass bei einer Übernahme durch das Parlament, die ja prinzipiell zu befürworten ist, die Andersdenkenden nicht mehr zur Artikulation qua Stimmabgabe kommen, liegt in der Natur dieser Verfahrensabkürzung. Jene Schwelle ist jedenfalls dann erreicht, wenn die Gegner des Anliegens aus dem Volksbegehren begründete Aussicht haben, an den Urnen zu siegen. Dann - und so war es in Brandenburg, wo nach den Umfragen bis zuletzt ein Vorsprung der Sachgegner vorhergesagt wurde - dürfen sie sich bei einer Übernahme mit Fug und Recht um die politische Durchsetzung ihrer Präferenz „geprellt“ fühlen.

Eine radikale Lösung, mit dieser Situation umzugehen, bestünde darin, auf das direkte Verfahren umzustellen, zumindest für das Volksbegehren. Dazu bräuchte man sich nicht gleich die Schweiz zum Vorbild zu nehmen, sondern könnte sich auch an das oben skizzierte sächsische Modell halten. Oder man könnte in die bestehenden Übernahmeklauseln der Landesverfassungen Vorbehalte im Sinne der eben formulierten Einschränkungen aufnehmen. Es würde aber bereits eine restriktive Interpretation helfen, die dem Ausnahmecharakter dieser Klauseln Rechnung trägt, die keineswegs einen Freibrief darstellen, um in das Verfahren der direkten Demokratie „hineinzufuhrwerken“. Institutionen und Verfahren haben ihre eigene Rationalität, ihren Sinn und ihre typischen Wirkungen. Sie begründen Erwartungen, Menschen verlassen sich auf sie und richten sich darauf ein. Der skizzierte Fall lässt vermuten, dass manche Verantwortlichen im repräsentativ-demokratischen System hierzulande immer noch Schwierigkeiten haben, mit der direkten Demokratie angemessen umzugehen; sie haben diese zwar eingeführt, können sie aber nicht „lassen“.

68 Eine Übersicht der Volksbegehren in Deutschland von 1945 bis 2008 von Otmar Jung findet sich bei Gunther Jürgens / Frank Rehmet, Direkte Demokratie in den Bundesländern - Ein Überblick, in: Hermann K. Heußner / Otmar Jung (Hrsg.), a.a.O. (Fn. 14), S. 197 - 233, S. 221 ff.; aufgenommen und fortgeschrieben bis 2009 von Frank Meerkamp, a.a.O. (Fn. 49), S. 542 f. Die seitdem zustande gekommenen Volksbegehren in Berlin 2010 mit 11,4 Prozent (Geheimverträge - Wasser, erforderlich waren sieben Prozent; vgl. Lars P. Feld / Peter M. Huber / Otmar Jung / Hans-Joachim Lauth / Fabian Wittreck (Hrsg.), Jahrbuch für direkte Demokratie 2011, Wiesbaden 2012, S. 132 f.), Brandenburg 2012 mit 5,0 Prozent (Nachtflugverbot, nötig waren 3,8 Prozent; vgl. dies. (Hrsg.), Jahrbuch für direkte Demokratie 2012, Wiesbaden 2013, im Erscheinen) und Bayern 2013 mit 14,3 Prozent (Studiengebühren, erforderlich waren zehn Prozent) fügen sich zu der obigen Aussage.

69 Insofern sieht der Hamburger Präzedenzfall von 2007 mit 8,2 Prozent Unterstützung eines Volksbegehrens (fünf Prozent waren nötig) auch nicht besonders gut aus.

70 Vgl. \$31 Abs. 1 Satz 2 LSA-VAbstG („Eintragungen, die über die erforderliche Unterschriftenzahl hinaus erfolgen, bleiben unberücksichtigt."), \$29 Abs. 2 Satz 3 ThürBVVG. 